\title{
Additive Lévy Processes: Capacity and Hausdorff Dimension
}

\author{
Davar Khoshnevisan and Yimin Xiao
}

\begin{abstract}
This is a survey on recently-developed potential theory of additive Lévy processes and its applications to fractal geometry of Lévy processes.

Additive Lévy processes arise naturally in the studies of the Brownian sheet, intersections of Lévy processes and so on. We first summarize some recent results on the novel connections between an additive Lévy process $X$ in $\mathbb{R}^{d}$, and a natural class of energy forms and their corresponding capacities. We then apply these results to study the Hausdorff dimension of the range and self-intersections of an ordinary Lévy process, solving several long-standing problems in the folklore of the theory of Lévy processes. We also list several open problems in this area.
\end{abstract}

Mathematics Subject Classification (2000). Primary 60J25, 28A80; Secondary 60G51, 60G17.

Keywords. Additive Lévy processes, Lévy sheets, Brownian sheet, capacity, Hausdorff dimension, multiple points.

\section{Introduction}

Classical potential theory has been very useful in studying the sample path properties of Markov processes, especially those of Brownian motion and Lévy processes. An important connection between classical potential theory and fractals is the fundamental result of Frostman (1935) [cf. Kahane (1985a) or Khoshnevisan (2002)] which states that the capacity dimension and the Hausdorff dimension [which will be denoted by $\left.\operatorname{dim}_{\mathrm{H}}\right]$ are the same. Hence, in order to prove $\operatorname{dim}_{\mathrm{H}} E \geq \beta$, it is sufficient to show that $E$ has positive capacity with respect to the Bessel-Riesz kernel $f(x)=\|x\|^{-\beta}$. Taylor $(1953,1955)$ applied this idea to determine the Hausdorff dimension of the range and graph of a Brownian motion in $\mathbb{R}^{d}$. Since then, this method has become one of the standard tools in obtaining lower bounds for

Research supported in part by a grant from the NSF. 
the Hausdorff dimension of random sets. We refer to the survey papers of Fristedt (1974), Taylor (1986) and Xiao (2003) for further information about the results and techniques for Lévy processes, and extensive lists of references.

There have been much effort in extending the potential theory for Markov processes to multiparameter processes of the forms $X(t)=X_{1}\left(t_{1}\right)+\cdots+X_{N}\left(t_{N}\right)$ or $Y(t)=\left(X_{1}\left(t_{1}\right), \ldots, X_{N}\left(t_{N}\right)\right)\left(t \in \mathbb{R}_{+}^{N}\right)$, where $X_{1}, \ldots, X_{N}$ are independent Markov processes; see e.g., Evans (1987a, b), Fitzsimmons and Salisbury (1989), Hirsch (1995), Hirsch and Song (1995a, b), Khoshnevisan (2002), Khoshnevisan and Xiao (2002, 2003a, b), Khoshnevisan, Xiao and Zhong (2003a). Besides of importance and interest in its own right, the potential theory of such multiparameter processes has found various applications in studying the fractal properties of ordinary Lévy processes, as well as their multiparameter analogue - the Lévy sheets.

The objective of this paper is to give a survey on the recently-developed potential theory of additive Lévy processes and its applications to fractal geometry of ordinary Lévy processes. In Section 2, we first recall the definition of Lévy processes and then introduce the definition of additive Lévy processes. We give two examples to show the close connections of additive Lévy processes to the Lévy sheets and ordinary Lévy processes. In Section 3, we summarize systematically the recent results on potential theory of additive Lévy processes. In Section 4, we show how the results in Section 3 can be applied to solve several long-standing problems about the ordinary Lévy processes. At the end of Sections 3 and 4, we list some open problems in the areas.

In the rest of this section, we give some general notation that will be used throughout. The underlying parameter space is $\mathbb{R}^{N}$, or $\mathbb{R}_{+}^{N}=[0, \infty)^{N}$. A typical parameter, $t \in \mathbb{R}^{N}$ is written as $t=\left(t_{1}, \ldots, t_{N}\right)$, sometimes also written as $\left\langle t_{j}\right\rangle$, or $\langle c\rangle$, if $t_{1}=\cdots=t_{N}=c \in \mathbb{R}$.

There is a natural partial order, "ß", on $\mathbb{R}^{N}$. Namely, $s \preccurlyeq t$ if and only if $s_{j} \leq t_{j}$ for all $j=1, \ldots, N$. When it is the case that $s \preccurlyeq t$, we define the interval, $[s, t]=\prod_{j=1}^{N}\left[s_{j}, t_{j}\right]$.

We will let $\mathcal{A}$ denote the class of all $N$-dimensional intervals $I \subset \mathbb{R}^{N}$ of the form $I=[s, t]$. We always write $\lambda_{m}$ for Lebesgue's measure on $\mathbb{R}^{m}$, no matter the value of the integer $m$. The state space, $\mathbb{R}^{d}$, is endowed with the $\ell^{2}$ Euclidean norm $\|\cdot\|$, and the corresponding dot product $\langle x, y\rangle=\sum_{j=1}^{d} x_{j} y_{j} \quad\left(x, y \in \mathbb{R}^{d}\right)$.

We write $\mathcal{P}(F)$ for the collection of all Borel-regular probability measures on a given Borel space $F$. Given a Borel measurable function $f: \mathbb{R}^{d} \rightarrow[0, \infty]$, we define the 'f-energy' (of some $\mu \in \mathcal{P}\left(\mathbb{R}^{d}\right)$ ) and ' $f$-capacity' (of some measurable $\left.G \subset \mathbb{R}^{d}\right)$ as follows:

$$
\mathcal{E}_{f}(\mu):=\iint f(x-y) \mu(d x) \mu(d y), \quad \mathcal{C}_{f}(G):=\left[\inf _{\mu \in \mathcal{P}(G)} \mathcal{E}_{f}(\mu)\right]^{-1} .
$$

We refer to such a function $f$ as a gauge function. 
Given a number $\beta>0$, we reserve $\mathcal{C}_{\beta}$ and $\mathcal{E}_{\beta}$ for $\mathcal{C}_{f}$ and $\mathcal{E}_{f}$ respectively, where the gauge function $f$ is $f(t):=\|t\|^{-\beta}$. $\mathcal{C}_{\beta}$ and $\mathcal{E}_{\beta}$ are respectively the ( $\beta$-dimensional) Bessel-Riesz capacity and energy to which some references were made earlier. More information about the Bessel-Riesz capacity and its connection to fractals can be found in Falconer (1990), Mattila (1995), Kahane (1985a), and Khoshnevisan (2002).

\section{Additive Lévy processes}

We first recall briefly the definition of a Lévy process and refer to Bertoin (1996) and Sato (1999) for the general theory. A stochastic process $Z=\{Z(t), t \geq 0\}$, with values in $\mathbb{R}^{d}$, is called a Lévy process, if it has stationary and independent increments, and such that $t \mapsto Z(t)$ is continuous in probability. It is well known that for $t \geq s \geq 0$, the characteristic function of $Z(t)-Z(s)$ is given by

$$
\mathbb{E}\left[e^{i\langle\xi, Z(t)-Z(s)\rangle}\right]=e^{-(t-s) \Psi(\xi)},
$$

where, by the Lévy-Khintchine formula,

$$
\Psi(\xi)=i\langle\mathrm{a}, \xi\rangle+\frac{1}{2}\left\langle\xi, \Sigma \xi^{\prime}\right\rangle+\int_{\mathbb{R}^{d}}\left[1-e^{i\langle x, \xi\rangle}+\frac{i\langle x, \xi\rangle}{1+\|x\|^{2}}\right] \mathrm{L}(d x), \quad \forall \xi \in \mathbb{R}^{d},
$$

and $a \in \mathbb{R}^{d}$ is fixed, $\Sigma$ is a non-negative definite, symmetric, $(d \times d)$ matrix, and $\mathrm{L}$ is a Borel measure on $\mathbb{R}^{d} \backslash\{0\}$ that satisfies

$$
\int_{\mathbb{R}^{d}} \frac{\|x\|^{2}}{1+\|x\|^{2}} \mathrm{~L}(d x)<\infty .
$$

The function $\Psi$ is the Lévy exponent of $Z$, and $\mathrm{L}$ is the corresponding Lévy measure. There are several different characterizations for the exponent $\Psi$. Note that $\Psi(0)=$ 0 and that by Bochner's theorem the function $\xi \mapsto e^{-t \Psi(\xi)}$ is continuous and positive definite for each $t \geq 0$ since it is the Fourier transform of a probability measure. Hence, the Lévy exponent $\Psi$ is a continuous negative definite function. See Berg and Frost (1975) for a systematic account on negative definite functions.

A Lévy process $Z$ is symmetric if $-Z$ and $Z$ have the same finite dimensional distributions. It is clear that $Z$ is symmetric if and only if $\Psi(\xi) \geq 0$, for all $\xi \in \mathbb{R}^{d}$. In particular, if the Lévy exponent $\Psi$ is of the form

$$
\Psi(\xi)=-\sigma^{\alpha}\|\xi\|^{\alpha} \quad \forall \xi \in \mathbb{R}^{d},
$$

where $\alpha \in(0,2]$ and $\sigma>0$ are constants, then $Z$ is called an isotropic stable Lévy process of index $\alpha$.

An $N$-parameter, $\mathbb{R}^{d}$-valued, additive Lévy process $X=\left\{X(t), t \in \mathbb{R}_{+}^{N}\right\}$ is a multiparameter stochastic process defined by

$$
X(t)=\sum_{j=1}^{N} X_{j}\left(t_{j}\right), \quad t \in \mathbb{R}_{+}^{N},
$$


where $X_{1}, \ldots, X_{N}$ denote independent Lévy processes that take their values in $\mathbb{R}^{d}$ and $X_{j}(0)=0$ for $j=1, \ldots, N$. Using tensor notation, we will often write $X=X_{1} \oplus \cdots \oplus X_{N}$ for brevity. If $X_{j}$ has Lévy exponent $\Psi_{j}$, then $\Psi=\left(\Psi_{1}, \ldots, \Psi_{N}\right)$ is called the Lévy exponent of $X$. When $X_{1}, \ldots, X_{N}$ are isotropic stable processes in $\mathbb{R}^{d}$ of index $\alpha \in(0,2]$, then $X$ is called an additive stable process. The case $\alpha=2$ yields the additive Brownian motion. Similarly, one can define an multiplicative Lévy process $X=X_{1} \otimes \cdots \otimes X_{N}$ by $X(t)=\left(X_{1}\left(t_{1}\right), \ldots, X_{N}\left(t_{N}\right)\right)$.

In the following we give two examples to show that additive Lévy processes arise naturally in the analysis of multiparameter processes such as Lévy's sheets and in the analysis of ordinary Lévy processes. As such, the potential theory of additive Lévy processes can also be used as a powerful tool to study the Hausdorff dimension and capacity of random fractals determined by Lévy processes.

Example 2.1 [The Lévy sheets and Brownian sheet] A Lévy sheet is a multiparameter extension of an ordinary Lévy process. For any given negative definite function $\Psi$ in $\mathbb{R}^{d}$, there is a random field $Z=\left\{Z(t), t \in \mathbb{R}_{+}^{N}\right\}$ taking values in $\mathbb{R}^{d}$ such that

(i) for any choice of disjoint intervals $Q_{j} \in \mathcal{A}(n=1, \ldots, n)$, the increments $Z\left(Q_{1}\right), \ldots, Z\left(Q_{n}\right)$ are independent; and

(ii) for each $Q \in \mathcal{A}$, the characteristic function of $Z(Q)$ is given by

$$
\mathbb{E}[\exp (i\langle\xi, Z(Q)\rangle)]=\exp \left(-\lambda_{N}(Q) \Psi(\xi)\right),
$$

where $Z(Q)$ is the increment of $Z$ on $Q=[s, t]$ which is defined by

$$
Z(Q)=\sum_{r \in\{0,1\}^{N}}(-1)^{N-\sum_{\ell} r_{\ell}} Z\left(\left\langle s_{j}+r_{j}\left(t_{j}-s_{j}\right)\right\rangle\right) .
$$

A random field $Z=\left\{Z(t), t \in \mathbb{R}_{+}^{N}\right\}$ taking values in $\mathbb{R}^{d}$ that is continuous in probability and satisfies the above conditions (i) and (ii) is called an $(N, d)$ Lévy sheet with exponent $\Psi$. Some general properties of Lévy sheets can be found in Adler et al. (1983), Dalang and Walsh (1992), as well as in Ehm (1981) and Vares (1983). When the Lévy exponent $\Psi$ is given by (2.1), $Z$ is called an isotropic stable sheet. In particular, when $\alpha=2$ and $\sigma=1 / \sqrt{2}$, then $Z$ is the $N$-parameter Brownian sheet in $\mathbb{R}^{d}$ and is usually denoted by $W=\left\{W(t), t \in \mathbb{R}_{+}^{N}\right\}$. We should mention that the Brownian sheet arises naturally in the stochastic partial differential equations and in statistical analysis [cf. Walsh (1986), Dalang (2003)] and is one of the most fundamental Gaussian random fields. By (2.1), we see that the components $W_{1}, \ldots, W_{d}$ of $W$ are independent, centered Gaussian random fields in $\mathbb{R}$ with the covariance function $\mathbb{E}\left[W_{1}(s) W_{1}(t)\right]=\prod_{j=1}^{N} s_{j} \wedge t_{j}$, where $a \wedge b=\min \{a, b\}$.

It follows from the conditions (i) and (ii) that a Lévy sheet $Z$ has independent and stationary increments. Along lines which are parallel to the axes, $Z$ is an ordinary Lévy process in $\mathbb{R}^{d}$ with a constant speed. More precisely, for any fixed $a=\left\langle a_{j}\right\rangle \in \mathbb{R}_{+}^{N}$ and every $k=1, \ldots, N$, the process $X_{k}=\left\{X_{k}\left(t_{k}\right), t_{k} \in \mathbb{R}_{+}\right\}$ 
defined by

$$
X_{k}\left(t_{k}\right)=Z\left(a_{1}, \ldots, a_{k-1}, a_{k}+t_{k}, a_{k+1}, \ldots, a_{N}\right)-Z(a)
$$

is a Lévy process in $\mathbb{R}^{d}$ with exponent $\Psi_{k}(\xi)=\left(\prod_{j \neq k} a_{j}\right) \Psi(\xi)$. This leads to the following very useful decomposition of $Z$ :

$$
Z(a+t)=Z(a)+\sum_{k=1}^{N} X_{k}\left(t_{k}\right)+\tilde{Z}(t), \quad t \in \mathbb{R}_{+}^{N},
$$

where $\tilde{Z}$ is a Lévy sheet in $\mathbb{R}^{d}$ with exponent $\Psi$, and all the processes on the right hand side of (2.4) are independent.

For many purposes such as in the studies of fractal properties of $Z$, the effects of $\tilde{Z}$ can sometimes be neglected. Hence the Lévy sheet $Z$ can be approximated locally by the additive Lévy process $X=X_{1} \oplus \cdots \oplus X_{N}$ which is easier to analyze. This approach has been exploited by Ehm (1981), Vares (1983), Rosen (1984), Zhong and Xiao (1995), Khoshnevisan, Xiao and Zhong (2003b) to study the local times and self-intersection local times of Lévy sheets; by Dalang and Mountford (1996, 1997, 2001, 2002, 2003), Dalang and Walsh (1993a, b) and Khoshnevisan (1995) to study the level sets, excursions and points of increase of the Brownian sheets [see also Dalang (2003)]; and by Khoshnevisan and Shi (1999), Khoshnevisan (1999) to establish the connection between hitting probabilities of the Brownian sheet and the Bessel-Riesz capacity.

Example 2.2 [Intersections of independent Lévy processes] Let $X_{1}, \ldots, X_{k}$ be independent Lévy processes in $\mathbb{R}^{d}$. It has been of interest to study whether their sample paths can intersect and, when they do, the fractal properties of the set of intersections; see Taylor (1986a), Xiao (2003) for further information. We say that the sample paths of $X_{1}, \ldots, X_{k}$ intersect if

$\exists$ distinct points $t_{1}, \ldots, t_{k} \in \mathbb{R}_{+}$such that $X_{1}\left(t_{1}\right)=\cdots=X_{k}\left(t_{k}\right)$.

Define the multiparameter process $Z=\left\{Z(t), t \in \mathbb{R}_{+}^{k}\right\}$ by

$$
Z(t)=\left(X_{2}\left(t_{2}\right)-X_{1}\left(t_{1}\right), \ldots, X_{k}\left(t_{k}\right)-X_{k-1}\left(t_{k-1}\right)\right) .
$$

Then (2.5) is equivalent to $Z^{-1}(0) \neq \emptyset$, where $Z^{-1}(0)=\left\{t \in \mathbb{R}_{+}^{k}: Z(t)=0\right\}$ is the zero set of $Z$. Rearranging the components of $Z$, it is easy to see that $Z$ is an additive Lévy process with values in $\mathbb{R}^{(k-1) d}$. Hence the potential theory of additive Lévy processes can be applied; see Theorem 3.6 below.

\section{Potential theory for additive Lévy processes}

Potential theory of multiparameter Markov processes have been studied by Evans (1987b), Fitzsimmons and Salisbury (1989) for multiplicative Markov processes and by Hirsch (1995), Hirsch and Song (1995a, b) [see also the recent book of Khoshnevisan (2002) for a systematic account on the earlier results], Khoshnevisan 
and Xiao (2002, 2003a, b), Khoshnevisan, Xiao and Zhong (2003a) for additive Lévy processes in $\mathbb{R}^{d}$.

The following are some natural questions that the potential theory of $X$ seeks to answer.

(a) Given a Borel set $F \subseteq \mathbb{R}^{d}$, when can $\mathbb{P}\left\{X\left(\mathbb{R}_{+}^{N}\right) \cap F \neq \emptyset\right\}>0$ ?

(b) For any fixed $a \in \mathbb{R}^{d}$ and a Borel set $E \subset \mathbb{R}_{+}^{N}$, when can $\mathbb{P}\left\{X^{-1}(a) \cap E \neq\right.$ $\emptyset\}>0$ ?

(c) When can the range of $X, X\left(\mathbb{R}_{+}^{N}\right)$ [or more generally $X(E)$, where $E \subset \mathbb{R}_{+}^{N}$ ], have positive Lebesgue measure?

For additive Lévy processes, the following lemma from Khoshnevisan and Xiao (2003b) shows that the above questions are closely related. For $N=1$, it is due to Kahane (1972).

Lemma 3.1. Let $X$ be an $N$-parameter additive Lévy process in $\mathbb{R}^{d}$. We assume that, for every $t \in(0, \infty)^{N}$, the distribution of $X(t)$ is mutually absolutely continuous with respect to $\lambda_{d}$. Then for any Borel sets $E \subset(0, \infty)^{N}$ and $F \subset \mathbb{R}^{d}$, the following are equivalent:

1. With positive probability, $E \cap X^{-1}(F) \neq \emptyset$;

2. With positive probability, $F \cap X(E) \neq \emptyset$;

3. With positive probability, $\lambda_{d}(F \ominus X(E))>0$, where $A \ominus B:=\{x-y: x \in A, y \in B\}$.

Under some mild conditions, Questions (a), (b) and (c) are answered in Khoshnevisan, Xiao and Zhong (2003a), and Khoshnevisan and Xiao (2002, 2003a, b). In this section, we summarize some of their results.

Let $X$ be an additive Lévy process in $\mathbb{R}^{d}$ with Lévy exponent $\Psi=\left(\Psi_{1}, \ldots, \Psi_{N}\right)$. It induces an energy form $\mathcal{E}_{\Psi}$ that can be described as follows: For all finite measures $\mu$ on $\mathbb{R}^{d}$, and/or all integrable functions $\mu: \mathbb{R}^{d} \rightarrow \mathbb{R}$,

$$
\varepsilon_{\Psi}(\mu)=(2 \pi)^{-d} \int_{\mathbb{R}^{d}}|\widehat{\mu}(\xi)|^{2} \prod_{j=1}^{N} \operatorname{Re}\left(\frac{1}{1+\Psi_{j}(\xi)}\right) d \xi,
$$

where ${ }^{\widehat{A}}$ denotes the Fourier transform normalized as $\widehat{f}(\xi)=\int_{\mathbb{R}_{d}} e^{i \xi \cdot x} f(x) d x[f \in$ $\left.L^{1}\left(\mathbb{R}^{d}\right)\right]$

Under the assumption that there exists a positive constant $\vartheta>0$ such that

$$
\operatorname{Re}\left(\prod_{j=1}^{N} \frac{1}{1+\Psi_{j}(\xi)}\right) \geq \vartheta \prod_{j=1}^{N} \operatorname{Re}\left(\frac{1}{1+\Psi_{j}(\xi)}\right),
$$

Khoshnevisan, Xiao and Zhong (2003a) have proved the following theorem.

Theorem 3.2. Consider any d-dimensional additive Lévy process $X$, whose Lévy exponent $\Psi$ satisfies (3.2). Then, given any nonrandom compact set $F \subset \mathbb{R}^{d}$, $\mathbb{E}\left\{\lambda_{d}\left(X\left(\mathbb{R}_{+}^{N}\right) \oplus F\right)\right\}>0$ if and only if $F$ carries a finite measure of finite energy. 
The proof of Theorem 3.2 consists of two parts. The sufficiency is established by using the Paley-Zygmund inequality [cf. Kahane (1985a,), p.8] and second moment estimates of the occupation measure of $X$. However the proof of the necessity is much harder and relies on developing a kind of multiparameter martingale theory in measure-spaces where the underlying measure has infinite mass.

By letting $F=\{0\}$, Theorem 3.2 gives the following answer to Question (c) for $X\left(\mathbb{R}_{+}^{N}\right)$, which extends the remarkable results of Kesten (1969) and Bretagnolle (1971) for $N=1$.

Corollary 3.3. Let $X$ be an additive Lévy process in $\mathbb{R}^{d}$ with Lévy exponent $\left(\Psi_{1}, \ldots, \Psi_{N}\right)$, and suppose that Condition (3.2) holds. Then,

$$
\mathbb{E}\left\{\lambda_{d}\left(X\left(\mathbb{R}_{+}^{N}\right)\right)\right\}>0 \Longleftrightarrow \int_{\mathbb{R}^{d}} \prod_{j=1}^{N} \operatorname{Re}\left(\frac{1}{1+\Psi_{j}(\xi)}\right) d \xi<+\infty .
$$

It is known that the positiveness of $\mathbb{E}\left\{\lambda_{d}\left(X\left(\mathbb{R}_{+}^{N}\right)\right)\right\}$ is closely related to the existence of local times of $X$. The precise connection for additive Lévy processes has been established by Khoshnevisan, Xiao and Zhong (2003b): under the condition $(3.2)$,

$$
X \text { has local times if and only if } \mathbb{E}\left\{\lambda_{d}\left(X\left(\mathbb{R}_{+}^{N}\right)\right)\right\}>0 \text {. }
$$

They have also proved results on the joint continuity, local and uniform Hölder conditions for the local times of additive Lévy processes, extending the results of Ehm (1981) and Vares (1983) for Lévy sheets.

More generally, for every compact $E \subset \mathbb{R}_{+}$, a necessary and sufficient condition in terms of the Lévy exponent $\left(\Psi_{1}, \ldots, \Psi_{N}\right)$ for $\mathbb{E}\left\{\lambda_{d}(X(E))\right\}>0$ has been given by Theorem 2.1 in Khoshnevisan, Xiao and Zhong (2003a). A different condition in terms of the gauge function is given by Theorem 3.6 below.

Applying Lemma 3.1 and Theorem 3.2 to additive stable Lévy processes, we have the following result which improves the earlier results of Hirsch (1995), Hirsch and Song (1995a, b), Khoshnevisan (2002).

Corollary 3.4. Suppose $X=X_{1} \oplus \cdots \oplus X_{N}$ is an additive stable process in $\mathbb{R}^{d}$ of index $\alpha \in(0,2]$. Then for any Borel set $F \subset \mathbb{R}^{d}$, the following are equivalent:

(i) $\mathrm{C}_{d-N \alpha}(F)>0$;

(ii) $\mathbb{P}\left\{\lambda_{d}\left\{F \oplus X\left(\mathbb{R}_{+}^{N}\right)\right\}>0\right\}>0$;

(iii) $F$ is not polar for $X$ in the sense that $\mathbb{P}\left\{F \cap X\left(\mathbb{R}_{+}^{N}\right) \neq \emptyset\right\}>0$.

Remark 3.5. Upon varying $d, N \in \mathbb{N}$, and $\alpha \in(0,2]$, we see that this theorem associates an additive Lévy process to any Bessel-Riesz capacity, including those with dimension $>2$. This connection is very useful in calculating the Hausdorff dimension of the range $X\left(\mathbb{R}_{+}^{N}\right)$ and various random fractals related to Lévy processes, via the co-dimension argument; see Section 4.

Note that, under the assumptions of Lemma 3.1, the conditions (3.2) and $\prod_{j=1}^{N} \operatorname{Re}\left(1+\Psi_{j}(\xi)\right)^{-1} \in L^{1}\left(\mathbb{R}^{d}\right)$ imply that $X$ hits points, i.e. $\mathbb{P}\left\{X^{-1}(a) \neq \emptyset\right\}>0$ 
for all $a \in \mathbb{R}^{d}$. In this case, it is natural to consider Question (b) and study the hitting probability of the level set $X^{-1}(a)$ for a fixed $a \in \mathbb{R}^{d}$. Moreover, when $X$ satisfies the conditions of Lemma 3.1, Question (b) is equivalent to the question (c) for $X(E)$. This latter problem for Brownian motion was considered by Hawkes (1977) and Kahane (1983, 1985a, b), and for additive Lévy processes by Khoshnevisan, Xiao and Zhong (2003a, Theorem 2.1). For symmetric additive Lévy processes, Question (b) is answered by the following theorem of Khoshnevisan and Xiao $(2002,2003 \mathrm{a})$. Recall that the additive Lévy process $X$ is absolutely continuous if for each $t \in(0, \infty)^{N}$, the function $\xi \mapsto \exp \left\{-\sum_{j=1}^{N} t_{j} \Psi_{j}(\xi)\right\} \in$ $L^{1}\left(\mathbb{R}^{d}\right)$. In this case, for every $t \in(0, \infty)^{N}, X(t)$ has a density function $p(t ; \bullet)$ that is given by the formula

$$
p(t ; x)=(2 \pi)^{-d} \int_{\mathbb{R}^{d}} e^{-i\langle\xi, x\rangle} \exp \left(-\sum_{j=1}^{N} t_{j} \Psi_{j}(\xi)\right) d \xi, \quad x \in \mathbb{R}^{d} .
$$

The function $\Phi$ defined by

$$
\Phi(s)=p(\bar{s}, 0) \quad s \in \mathbb{R}^{N},
$$

where $\bar{s}=\left\langle\left|s_{j}\right|\right\rangle \in \mathbb{R}_{+}^{N}$, is called the gauge function for $X$. Khoshnevisan and Xiao (2002, Theorem 2.9) have shown that $\Phi \in L_{l o c}^{1}\left(\mathbb{R}^{N}\right)$ is equivalent to $X^{-1}(a) \neq \emptyset$.

Theorem 3.6. Let $X_{1}, \ldots, X_{N}$ be $N$ independent symmetric Lévy processes on $\mathbb{R}^{d}$ and let $X=X_{1} \oplus \cdots \oplus X_{N}$. Suppose $X$ is absolutely continuous with the gauge function $\Phi$. Then for all $a \in \mathbb{R}^{d}, c>0$ and for all compact sets $E \subset\left[c, \infty\left[{ }^{N}\right.\right.$,

$$
\begin{gathered}
A_{1} \mathcal{C}_{\Phi}(E) \leq \mathbb{P}\left\{X^{-1}(a) \cap E \neq \emptyset\right\} \leq A_{2} \mathcal{C}_{\Phi}(E) \text {, where } \\
A_{1}=\kappa^{-2} 2^{-d}\{\Phi(\langle c\rangle)\}^{-1} I_{E}^{2}(a), A_{2}=\kappa^{3} 2^{5 d+3 N} \Phi(\langle c\rangle) \text { and } I_{E}(a)=\inf _{s \in E} p(s ; a) .
\end{gathered}
$$

Theorem 2.10 in Khoshnevisan and Xiao (2002) also gives a formula for $\operatorname{dim}_{\mathrm{H}} X^{-1}(0)$. In particular, when $X$ is an additive stable process, we have

Corollary 3.7. Suppose $X_{1}, \ldots, X_{N}$ are independent isotropic stable Lévy processes in $\mathbb{R}^{d}$ with index $\left.\left.\alpha \in\right] 0,2\right]$ and $X=X_{1} \oplus \cdots \oplus X_{N}$. Then,

(i) $\mathbb{P}\left\{X^{-1}(0) \neq \varnothing\right\}>0$ if and only if $N \alpha>d$; and

(ii) if $N \alpha>d$, then $\mathbb{P}\left\{\operatorname{dim}_{\mathrm{H}} X^{-1}(0)=N-\frac{d}{\alpha}\right\}=1$.

Furthermore, for each $M>1$, there exists a constant $A>1$, such that simultaneously for all compact sets $E \subset\left[M^{-1}, M\right]^{N}$, and for all $a \in[-M, M]^{d}$,

$$
\frac{1}{A} \mathcal{C}_{d / \alpha}(E) \leq \mathbb{P}\left\{X^{-1}(a) \cap E\right\} \leq A \mathcal{C}_{d / \alpha}(E)
$$

Remark 3.8. In Theorem 1.1 of Khoshnevisan and Xiao (2002), it is proved that (ii) in Corollary 3.7 holds with positive probability. It can be strengthened to a probability 1 result by using the conditional Borel-Cantelli lemma.

There are several interesting open questions in this area. In the following, we list some of them. 
Problem 3.9. Can the condition (3.2) be dropped from Theorem 3.2?

A positive resolution to this question would lead to the following:

(i) A complete resolution of a conjecture regarding intersection of independent regenerative sets of J. Bertoin (1999, p. 49).

(ii) The computation of the Hausdorff dimension of the range of an arbitrary additive Lévy process; see Theorem 4.1 below for a partial result.

A resolution of item (ii) would go a long way toward solving the corresponding open problem for an arbitrary Lévy sheet (Straf 1972; Ehm 1981; Vares 1983).

Problem 3.10. Can the symmetry and absolute continuity assumptions be dropped from Theorem 3.6?

A positive solution to Problem 3.10 will have interesting consequences on the intersections of Lévy processes and on the Hausdorff dimension of the multiple points.

Another natural question is to characterize the polar sets for the space-time process $\left\{(t, X(t)) ; t \in \mathbb{R}_{+}^{N}\right\}$ :

Problem 3.11. Given Borel sets $E \subseteq \mathbb{R}_{+}^{N}$ and $F \subseteq \mathbb{R}^{d} \backslash\{0\}$, find a necessary and sufficient condition for $\mathbb{P}\{X(E) \cap F \neq \emptyset\}>0$ ?

In the case that $N=1$ and $X$ is a $d$-dimensional Brownian motion, the above problem is equivalent to the polarity of the set $E \times F$ relative to the Dirichlet problem for the full heat operator $\partial_{t}-\frac{1}{2} \Delta$; see Doob $(1984$, p. 637) and Watson $(1976,1978)$. In particular, Watson has proven that a set $G \subset \mathbb{R}_{+} \times \mathbb{R}^{d}$ is polar for the heat equation if and only if it has zero thermal capacity. See also Kaufman and $\mathrm{Wu}(1982)$ and Taylor and Watson (1985) for further information.

A resolution of Problem 3.11 will contribute to developing a complete parabolic potential theory for various multiparameter Lévy processes. This is not only interesting of itself, but will also solve a number of old problems in the theory of one-parameter Markov processes (including one-dimensional Brownian motion). For instance, problems about the Hausdorff dimension of the set $B(E) \cap F$, where $E$ and $F$ are, respectively, compact subsets of $\mathbb{R}_{+}$and $\mathbb{R}^{d} \backslash\{0\}$. This later problem was first considered by Kaufman (1972), who obtained upper and lower bounds. Hawkes (1978a) has found an extension of Kaufman's theorem to stable processes. However, in general, the Hausdorff dimension of $B(E) \cap F$ is unknown even for Brownian motion.

We remark that Problem 3.11 is intimately related to the existing potential theory of additive Lévy processes. Indeed, in Khoshnevisan and Xiao (2002, 2003a), we find a necessary and sufficient condition for $\mathbb{P}\left\{X(E) \cap\left\{x_{0}\right\} \neq \emptyset\right\}$ to be positive for an arbitrary symmetric additive Lévy process $X$ that has a density for all $t>0$. On the other hand, Khoshnevisan, Xiao and Zhong (2003a) have derived a necessary and sufficient condition for $\mathbb{P}\left\{X\left(\mathbb{R}_{+}^{N}\right) \cap F \neq \emptyset\right\}$ to be positive; see also Khoshnevisan and Xiao (2003b). These results can all be considered as partial solutions to Problem 3.11. 
We believe that once Problem 3.11 is solved, the methods of Khoshnevisan and Xiao $(2002,2003 \mathrm{a}, 2003 \mathrm{~b})$ can be used to calculate the Hausdorff dimensions of the random sets $X(E) \cap F$ and $X^{-1}(F) \cap E$ not only for Brownian motion, but also for a large class of Lévy processes.

There are many other difficult open problems of similar parabolic nature in the literature. For example, let $F(\lambda)$ denote the set of $\lambda$-fast points of Brownian motion,

$$
F(\lambda)=\left\{t \in[0,1]: \limsup _{h \rightarrow 0+} \frac{|B(t+h)-B(t)|}{\sqrt{2 h|\log h|}} \geq \lambda\right\} .
$$

Then, given a Borel set $E \subset \mathbb{R}_{+}$, what is the Hausdorff dimension of the set $F(\lambda) \cap E$ ? See Orey and Taylor (1974), Kaufman (1975), Khoshnevisan, Peres and Xiao (2000), and Khoshnevisan and Shi (2000) for some information. The same question can be asked when $F(\lambda)$ denotes the set of thick points of the occupation measure of a Brownian motion in $\mathbb{R}^{d}$ (Dembo, Peres, Rosen and Zeitouni, 2000). We believe that a sufficiently powerful connection to parabolic potential theory may unravel some of these problems.

Finally, Khoshnevisan and Shi (1999) have proven that the $(N, d)$-Brownian sheet $W$ and the $(N, d)$-additive Brownian motion $B=B_{1} \oplus \cdots \oplus B_{N}$ are intersection equivalent, i.e. for any compact set $F \subset \mathbb{R}^{d}$,

$$
\mathbb{P}\left\{W\left(\mathbb{R}_{+}^{N}\right) \cap F \neq \emptyset\right\}>0 \Longleftrightarrow \mathbb{P}\left\{B\left(\mathbb{R}_{+}^{N}\right) \cap F \neq \emptyset\right\}>0,
$$

see Corollary 6.2 of Khoshnevisan and Shi (1999). It would be interesting to solve the analogous problem for the $(N, d)$-stable sheets [cf. Ehm (1981)]. In particular, we believe that

Conjecture 3.12. Corollary 3.4 holds for an $(N, d)$-stable sheet of type $A$.

\section{Applications to fractal properties of Lévy processes}

By Corollary 3.4 and the Frostman's theorem [cf. Kahane (1985, p.133) or Khoshnevisan $\left(2002\right.$, p.521)], we can use the range $X\left(\mathbb{R}_{+}^{N}\right)$ as a tool to determine the Hausdorff dimension of any Borel set $F$ in $\mathbb{R}^{d}$. The original idea goes back to Taylor (1966), who showed that for any Borel set $F \subset \mathbb{R}^{d}$ with $\operatorname{dim}_{\mathrm{H}} F \geq d-2$,

$$
\operatorname{dim}_{\mathrm{H}} F=d-\inf \left\{\alpha>0: F \text { is not polar for } X_{\alpha}\right\},
$$

where $X_{\alpha}$ is an isotropic stable Lévy process in $\mathbb{R}^{d}$ of index $\alpha \in(0,2]$. With the help of additive stable processes, the restriction on $F$ can be removed. Corollary 3.4 implies that for any Borel set $F \subset \mathbb{R}^{d}$,

$$
\operatorname{dim}_{\mathrm{H}} F=d-\inf \left\{N \alpha>0: F \text { is not polar for } X_{\alpha, N}\right\},
$$

where $X_{\alpha, N}$ denotes an $N$-parameter additive stable process in $\mathbb{R}^{d}$ of index $\alpha \in$ $(0,2]$. Following Khoshnevisan and Shi $(2000)$, this method for calculating $\operatorname{dim}_{\mathrm{H}} F$ is called the co-dimension argument, which is specially effective for determining the Hausdorff dimension of a random set. See Hawkes (1981), Lyons (1990), 
Peres (1996, 1999), Khoshnevisan, Peres and Xiao (2000), Khoshnevisan (2002) for related results.

\subsection{Hausdorff dimension of the range}

As an application of the co-dimension argument, we derive the Hausdorff dimension of the range of an additive Lévy process, which is proved in Khoshnevisan, Xiao and Zhong (2003a).

Theorem 4.1. Given an additive Lévy process $X$ in $\mathbb{R}^{d}$ with Lévy exponent $\left(\Psi_{1}, \ldots, \Psi_{N}\right)$ that satisfies $(3.2)$,

$$
\operatorname{dim}_{\mathrm{H}}\left(X\left(\mathbb{R}_{+}^{N}\right)\right)=d-\eta, \quad \mathbb{P} \text {-a.s. }
$$

where

$$
\eta=\sup \left\{\gamma>0: \int_{\xi \in \mathbb{R}^{d}:\|\xi\|>1} \prod_{j=1}^{N} \operatorname{Re}\left(\frac{1}{1+\Psi_{j}(\xi)}\right) \frac{d \xi}{\|\xi\|^{\gamma}}=+\infty\right\} .
$$

Here $\sup \emptyset=0$. In particular, if $X$ is an additive stable process in $\mathbb{R}^{d}$ of index $\alpha \in(0,2]$, then $\operatorname{dim}_{\mathrm{H}} X\left(\mathbb{R}_{+}^{N}\right)=\min \{d, \alpha N\}$ a.s.

Sketch of Proof. We introduce an $M$-parameter additive stable process $Y$ in $\mathbb{R}^{d}$ with index $\alpha \in(0,2]$. The process $Y$ is totally independent of $X$, and the constants $M$ and $\alpha$ will be chosen appropriately. Note that $X \oplus Y$ is an $(N+M)$-parameter additive Lévy process in $\mathbb{R}^{d}$ whose Lévy exponent $\Phi=\left(\Phi_{1}, \ldots, \Phi_{N+M}\right)$ is given by

$$
\Phi_{j}(\xi)= \begin{cases}\Psi_{j}(\xi), & \text { if } j=1, \ldots, N \\ \frac{1}{2}\|\xi\|^{\alpha}, & \text { if } j=N+1, \ldots, N+M\end{cases}
$$

Then by Theorem 3.2, Corollary 3.4 and the zero-one law that $\mathbb{P}\left\{\complement_{\beta}\left(X\left(\mathbb{R}_{+}^{N}\right)\right)>\right.$ $0\} \in\{0,1\}$ for any $\beta>0$ [whose proof will be given elsewhere], we have

$$
\mathcal{C}_{d-M \alpha}\left(X\left(\mathbb{R}_{+}^{N}\right)\right)>0 \text { a.s. } \Longleftrightarrow \int_{\xi \in \mathbb{R}^{d}:\|\xi\|>1} \prod_{j=1}^{N} \operatorname{Re}\left(\frac{1}{1+\Psi_{j}(\xi)}\right)\|\xi\|^{-M \alpha} d \xi<\infty .
$$

From this follows (4.1).

When $N=1$, i.e., when $X$ is an ordinary Lévy process in $\mathbb{R}^{d}$, Pruitt (1969) has shown that the Hausdorff dimension of the range $X\left(\mathbb{R}_{+}\right)$is

$$
\gamma=\sup \left\{\alpha \geq 0: \limsup _{r \rightarrow 0} r^{-\alpha} \int_{0}^{1} \mathbb{P}\{|X(t)| \leq r\} d t<+\infty\right\} .
$$

In general, this formula is not satisfying, since the above limsup is not easy to evaluate. Pruitt (1969, Theorem 5) addresses this issue by showing that if $\operatorname{Re} \Psi(\xi) \geq 2 \log \|\xi\|$ (for all $\|\xi\|$ large), then,

$$
\gamma=\sup \left\{\alpha<d: \int_{\mathbb{R}^{d}} \operatorname{Re}\left(\frac{1-e^{-\Psi(\xi)}}{\Psi(\xi)}\right) \frac{d \xi}{\|\xi\|^{d-\alpha}}<+\infty\right\} .
$$


See Fristedt $(1974$, 377-378) for further discussions on Pruitt's work in this area.

Theorem 4.1 implies the following representation for the index $\gamma$ in terms of the Lévy exponent $\Psi$ of $X$ that holds under no restrictions, thus solving the above mentioned problem of Pruitt (1969).

Corollary 4.2. If $X$ denotes a Lévy process in $\mathbb{R}^{d}$ with Lévy exponent $\Psi$, then

$$
\operatorname{dim}_{\mathrm{H}} X([0,1])=\sup \left\{\alpha<d: \int_{\xi \in \mathbb{R}^{d}:\|\xi\|>1} \operatorname{Re}\left(\frac{1}{1+\Psi(\xi)}\right) \frac{d \xi}{\|\xi\|^{d-\alpha}}<+\infty\right\} .
$$

Formula (4.3) gives an analytic way to compute the Hausdorff dimension of $X\left(\mathbb{R}_{+}\right)$for Lévy processes. Its usefulness is shown by the following result from Khoshnevisan and Xiao (2003b).

Corollary 4.3. Let $X=\left\{X(t), t \in \mathbb{R}_{+}\right\}$be a Lévy process in $\mathbb{R}^{d}$ with Lévy exponent $\Psi$. If $\Psi$ satisfies the following condition: for any $\epsilon>0$, there exist positive constants $K_{1}$ and $K_{2}$ such that

$\frac{K_{1}}{\sum_{j=1}^{d}\left|\xi_{j}\right|^{\alpha_{j}+\epsilon}} \leq \operatorname{Re}\left(\frac{1}{1+\Psi(\xi)}\right) \leq \frac{K_{2}}{\sum_{j=1}^{d}\left|\xi_{j}\right|^{\alpha_{j}-\epsilon}}, \quad \forall \xi \in \mathbb{R}^{d}$ with $\min _{1 \leq j \leq d}\left|\xi_{j}\right| \geq 1$,

then almost surely,

$$
\operatorname{dim}_{\mathrm{H}} X\left(\mathbb{R}_{+}\right)= \begin{cases}\alpha_{1} & \text { if } \alpha_{1} \leq d_{1}, \\ 1+\alpha_{2}\left(1-1 / \alpha_{1}\right) & \text { otherwise. }\end{cases}
$$

It is easily seen that the Lévy processes with independent stable components [cf. Hendricks $(1973,1974)]$ satisfy (4.4). Meerchaert and Xiao (2003) have recently shown that every operator stable Lévy process $X$ in $\mathbb{R}^{d}$ with exponent $B$ satisfies (4.4) with $\alpha_{1}, \ldots, \alpha_{d}$ being the real parts of the eigenvalues of $B$. Hence the Hausdorff dimension of the range $X\left(\mathbb{R}_{+}\right)$is given by (4.5). This solves a problem of Becker-Kern, Meerschaert and Scheffler (2003).

When $X$ is a Brownian motion or a stable Lévy process in $\mathbb{R}^{d}$, McKean (1955) and Blumenthal and Getoor (1960) have demonstrated that for all Borel sets $E \subset \mathbb{R}_{+}$,

$$
\operatorname{dim}_{\mathrm{H}} X(E)=d \wedge \alpha \operatorname{dim}_{\mathrm{H}} E, \quad \text { a.s., }
$$

Blumenthal and Getoor (1961) extended (4.6) to a broad class of Lévy processes. For this purpose, they introduced the upper index $\beta$ and lower indices $\beta^{\prime}, \beta^{\prime \prime}$ of a general Lévy process $X$ and, in addition, the lower index $\sigma$ of a subordinator. Blumenthal and Getoor (1961, Theorems 8.1 and 8.5) established the following upper and lower bounds for $\operatorname{dim}_{\mathrm{H}} X(E)$ in terms of the upper index $\beta$ and lower indices $\beta^{\prime}$ and $\beta^{\prime \prime}$ of $X$ : For every $E \subset \mathbb{R}_{+}$, almost surely

$$
\operatorname{dim}_{\mathrm{H}} X(E) \leq \beta \operatorname{dim}_{\mathrm{H}} E, \quad \text { if } \beta<1,
$$

and

$$
\operatorname{dim}_{\mathrm{H}} X(E) \geq \begin{cases}\beta^{\prime} \operatorname{dim}_{\mathrm{H}} E, & \text { if } \beta^{\prime} \leq d, \\ 1 \wedge \beta^{\prime \prime} \operatorname{dim}_{\mathrm{H}} E, & \text { if } \beta^{\prime}>d=1 .\end{cases}
$$


They showed, in addition, that when $X$ is a subordinator, then

$$
\sigma \operatorname{dim}_{\mathrm{H}} E \leq \operatorname{dim}_{\mathrm{H}} X(E) \leq \beta \operatorname{dim}_{\mathrm{H}} E, \quad \text { a.s. }
$$

The restriction $\beta<1$ of (4.7) was removed subsequently by Millar (1971, Theorem $5.1)$. Blumenthal and Getoor (1961, p.512) conjectured that, given a Borel set $E \subset[0,1]$, there exists a constant $\lambda(X, E)$ such that

$$
\operatorname{dim}_{\mathrm{H}} X(E)=\lambda(X, E), \quad \text { a.s. }
$$

Except in the cases that $X$ is a Lévy process with stable components [Hendricks (1973)], or a subordinator [Hawkes (1978b)], the question whether the above conjecture holds or not had remained unanswered. Recently, by using potential of additive Lévy processes, Khoshnevisan and Xiao (2003b) have verified the above conjecture by proving the following theorem.

Theorem 4.4. Suppose $X=\left\{X(t) ; t \in \mathbb{R}_{+}\right\}$is a Lévy process in $\mathbb{R}^{d}$, and denote its Lévy exponent by $\Psi$. Then for any Borel set $E \subset \mathbb{R}_{+}$, a.s.

$$
\begin{aligned}
& \operatorname{dim}_{\mathrm{H}} X(E) \\
& =\sup \left\{\beta \in(0, d): \inf _{\mu \in \mathcal{P}(E)} \int_{\mathbb{R}^{d}} \iint e^{-|s-t| \Psi(\operatorname{sgn}(s-t) \xi)}\|\xi\|^{\beta-d} \mu(d s) \mu(d t) d \xi<+\infty\right\} .
\end{aligned}
$$

If $X$ is symmetric, then (4.10) is equivalent to the following:

$$
\operatorname{dim}_{\mathrm{H}} X(E)=\sup \left\{\beta \in(0, d): \mathcal{C}_{f_{d-\beta}}(E)>0\right\}, \quad \text { a.s., }
$$

where $f_{\gamma}$ is defined as

$$
f_{\gamma}(x):=\int_{\mathbb{R}^{d}} e^{-|x| \Psi(\xi)}\|\xi\|^{-\gamma} d \xi, \quad{ }^{\forall} x \in \mathbb{R}, \gamma \in(0, d) .
$$

Formula (4.10) shows that $\operatorname{dim}_{\mathrm{H}} X(E)$ may depend on other characteristics of the set $E$ than its Hausdorff dimension and it is not easy to use. Only in a few cases, we can express $\operatorname{dim}_{\mathrm{H}} X(E)$ in terms of $\operatorname{dim}_{\mathrm{H}} E$; see Blumenthal and Getoor (1960), Hendricks (1973), Meerchaert and Xiao (2003).

After the Hausdorff dimension of $X\left(\mathbb{R}_{+}\right)$or $X(E)$ is known, it is natural to study its exact Hausdorff measure or exact capacity. The former problem has been studied extensively; see Taylor (1986a) or Xiao (2003) for a survey. The latter problem has been investigated by Kahane (1985b) and Hawkes (1998) for symmetric stable Lévy processes and subordinators, respectively. Applying potential theory of additive Lévy processes, Khoshnevisan and Xiao (2003b) have recently extended their results to general Lévy processes.

On the other hand, Pemantle, Peres and Shapiro (1996) have shown that the range of Brownian motion $B$ in $\mathbb{R}^{d}(d \geq 3)$ is capacity equivalent to $[0,1]^{2}$. Rosen (2000) extends their results to a class of Lévy processes and introduces the notion of capacitary modulus. A function $h(x): \mathbb{R}^{d} \rightarrow \mathbb{R}_{+}$is called a capacitary modulus for $\Lambda \subset \mathbb{R}^{d}$ if there exist constants $0<K_{3} \leq K_{4}<\infty$ such that

$$
\left[K_{4} \int_{\mathbb{R}^{d}} f(|x|) h(x) d x\right]^{-1} \leq \mathcal{C}_{f}(\Lambda) \leq\left[K_{3} \int_{\mathbb{R}^{d}} f(|x|) h(x) d x\right]^{-1}
$$


for all non-increasing $f: \mathbb{R}_{+} \rightarrow[0, \infty]$. Thus, the results of Pemantle, Peres and Shapiro (1996) imply that the function

$$
h(x)= \begin{cases}|x|^{-(d-2)} & \text { if } d \geq 3 \\ |\log x| & \text { if } d=2\end{cases}
$$

is a capacity modulus for $B([0,1])$. It would be interesting to study capacitary modulus for the range of an additive Lévy processes.

\subsection{Existence and Hausdorff dimension of multiple points}

Let $X=\left\{X(t), t \in \mathbb{R}_{+}\right\}$be a $d$-dimensional Lévy process. The existence of $k$ multiple points of $X$ has been solved by LeGall et al. (1989), Evans (1987a), Fitzsimmons and Salisbury (1989). In this subsection, we show that the potential theory of additive Lévy processes can be applied to study the existence of selfintersections of $X(t)$ when $t$ is restricted to disjoint compact subsets $E_{1}, E_{2}, \ldots, E_{k}$ of $\mathbb{R}_{+}(k \geq 2)$. This problem was originally considered by Kahane (1983) who showed that if $X$ is a symmetric stable Lévy process in $\mathbb{R}^{d}$ of index $\alpha$ and $k=2$, then

$$
\mathcal{C}_{d / \alpha}\left(E_{1} \times E_{2}\right)>0 \Rightarrow \mathbb{P}\left\{X\left(E_{1}\right) \cap X\left(E_{2}\right) \neq \emptyset\right\}>0 \Rightarrow \lambda_{d / \alpha}\left(E_{1} \times E_{2}\right)>0 .
$$

Kahane $(1983$, p. 90$)$ conjectured that $\mathcal{C}_{d / \alpha}\left(E_{1} \times E_{2}\right)>0$ is necessary and sufficient for $\mathbb{P}\left\{X\left(E_{1}\right) \cap X\left(E_{2}\right) \neq \emptyset\right\}>0$. This conjecture was verified by Khoshnevisan (1999) for Brownian motion. The following theorem of Khoshnevisan and Xiao (2003b) establishes a necessary and sufficient condition for $\mathbb{P}\left\{X\left(E_{1}\right) \cap \cdots \cap X\left(E_{k}\right) \neq\right.$ $\emptyset\}>0$ and implies that Kahane's conjecture is true for all symmetric stable Lévy processes.

Theorem 4.5 (Kahane's Problem). Let $X$ be a symmetric Lévy process in $\mathbb{R}^{d}$ with Lévy exponent $\Psi$. If the distribution of $X(t)$ is equivalent to $\lambda_{d}$ for all $t>0$, then for any disjoint compact sets $E_{1}, \ldots, E_{k} \subset \mathbb{R}_{+}, \mathbb{P}\left\{X\left(E_{1}\right) \cap \cdots \cap X\left(E_{k}\right) \neq \emptyset\right\}>0$ if and only if $\mathcal{C}_{f}\left(E_{1} \times E_{2} \times \cdots \times E_{k}\right)>0$, where

$$
f(x):=\int_{\mathbb{R}^{d(k-1)}} \exp \left(-\sum_{j=1}^{k}\left|x_{j}\right| \Psi\left(\xi_{j-1}-\xi_{j}\right)\right) d \xi, \quad x \in \mathbb{R}^{k}, \xi_{0}=\xi_{k}=0 .
$$

To prove Theorem 4.5, it suffices to consider $k$ independent Lévy processes $X_{1}, \ldots, X_{k}$ in $\mathbb{R}^{d}$ with exponent $\Psi$ and define an additive Lévy process $Z$ by (2.6). Then by Lemma 3.1, we need to find a necessary and sufficient condition for $\mathbb{E}\left\{\lambda_{d(k-1)}\left(Z\left(E_{1} \times \cdots \times E_{k}\right)\right)\right\}>0$. This is done by Theorem 2.1 of Khoshnevisan, Xiao and Zhong (2003a) [see also Theorem 3.6, under some extra conditions].

Now we consider the fractal properties of the set $M_{k}^{(d)}$ of $k$-multiple points and the set $L_{k}^{(d)}$ of $k$-multiple times of $X$ defined by

$$
M_{k}^{(d)}=\left\{x \in \mathbb{R}^{d}: x=X\left(t_{1}\right)=\cdots=X\left(t_{k}\right) \text { for distinct } t_{1}, \ldots, t_{k} \in \mathbb{R}_{+}\right\}
$$

and

$$
L_{k}^{(d)}=\left\{\left(t_{1}, \cdots, t_{k}\right) \in \mathbb{R}_{+}^{k}, t_{1}, \ldots, t_{k} \text { are distinct and } X\left(t_{1}\right)=\cdots=X\left(t_{k}\right)\right\},
$$


respectively.

The Hausdorff dimension $\operatorname{dim}_{\mathrm{H}} M_{k}^{(d)}$ has been determined by Taylor (1966), Fristedt (1967), Hendricks (1974), Hawkes (1978c), Shieh (1998) for stable Lévy processes, operator stable Lévy processes and certain isotropic Lévy processes, respectively. However, the following problem is still open:

Problem 4.6. Given a general $d$-dimensional Lévy process $X$, compute $\operatorname{dim}_{\mathrm{H}} M_{k}^{(d)}$ and $\operatorname{dim}_{\mathrm{P}} M_{k}^{(d)}$ explicitly in terms of the Lévy exponent of $X$, where $\operatorname{dim}_{\mathrm{P}}$ denotes packing dimension.

Note that Corollary 4.2 computes the Hausdorff dimension of $M_{1}$. In order to understand the fine structure of $M_{k}$ for $k>1$, we need to appeal to the potential theory for multiplicative Lévy processes $X=X_{1} \otimes \cdots \otimes X_{k}$. Prefatory results, along these lines, have been developed earlier in Evans (1987a, b), Fitzsimmons and Salisbury (1989), Peres (1999), and Khoshnevisan (2002).

Very little is known about the packing dimension aspect of Problem 4.6, and completely new ideas are needed. For $k=1$, Taylor (1986b) has proven that $\operatorname{dim}_{\mathrm{P}} X\left(\mathbb{R}_{+}\right)$is equal to a certain exponent $\gamma^{\prime}$ a.s., but $\gamma^{\prime}$ seems, in general, incomputable. It would be desirable to represent $\gamma^{\prime}$ in terms of the Lévy exponent $\Psi$.

For the Hausdorff dimension of $L_{k}^{(d)}$, Rosen (1983) has shown that if $X$ is a $d$-dimensional Brownian motion $(d=2,3)$, then

$$
\operatorname{dim}_{\mathrm{H}} L_{2}^{(3)}=\frac{1}{2} \quad \text { and } \quad \operatorname{dim}_{\mathrm{H}} L_{k}^{(2)}=1 \text { for all } k \geq 2 .
$$

The following more general result is derived from Theorem 2.10 in Khoshnevisan and Xiao (2002) [see also Khoshnevisan and Xiao (2003a)]:

Theorem 4.7. If $X$ is a symmetric Lévy process in $\mathbb{R}^{d}$ with exponent $\Psi$ such that $\xi \mapsto e^{-t \Psi(\xi)}$ is in $L^{1}\left(\mathbb{R}^{d}\right)$. Then

$$
\operatorname{dim}_{\mathrm{H}} L_{k}^{(d)}=\sup \left\{b>0: \int_{[0,1]^{k}} \frac{1}{|\mathbf{s}|^{b}} \Phi(\mathbf{s}) d \mathbf{s}<\infty\right\},
$$

where $\Phi$ is the gauge function on $\mathbb{R}^{k}$ defined by

$$
\Phi(\mathbf{s})=(2 \pi)^{-d} \int_{\mathbb{R}^{(k-1) d}} \exp \left(-\sum_{j=1}^{k}\left|s_{j}\right| \Psi\left(\xi_{j}-\xi_{j-1}\right)\right) d \xi \quad \text { for } \mathbf{s}=\left\langle s_{j}\right\rangle \in \mathbb{R}^{k}
$$

In particular, if $X$ is a symmetric stable Lévy processes in $\mathbb{R}^{d}$ with index $\alpha \in(0,2]$ and such that $\alpha k>(k-1) d\left[\right.$ i.e., $\left.L_{k}^{(d)} \neq \emptyset\right]$, then

$$
\operatorname{dim}_{\mathrm{H}} L_{k}^{(d)}=k-\frac{(k-1) d}{\alpha} .
$$

This extends Rosen's result (4.14).

The conditions of Theorem 4.7 are quite restrictive. It would be interesting to solve the following: 
Problem 4.8. Find $\operatorname{dim}_{\mathrm{H}} L_{k}^{(d)}$ and $\operatorname{dim}_{\mathrm{P}} L_{k}^{(d)}$ for a general Lévy process $X$ in $\mathbb{R}^{d}$.

Except for Brownian motion, there is no knowledge on the exact Hausdorff and packing measure functions of $M_{k}$ and $L_{k}$. These problems have to be studied by using other methods than potential theory of additive Lévy processes, and hence will not be discussed here. We refer to Xiao (2003b, section 9) for more information.

\section{References}

[1] R. J. Adler (1981), The Geometry of Random Fields. Wiley, New York.

[2] R. J. Adler, D. Monrad, R. H. Scissors and R. Wilson (1981), Representation, decompositions and sample function continuity of random fields with independent increments. Stoch. Proc. Appl. 15, 3-30.

[3] P. Becker-Kern, M. M. Meerschaert and H. P. Scheffler (2003), Hausdorff dimension of operator stable sample paths. Monatsh. Math. to appear.

[4] C. Berg and G. Forst (1975), Potential Theory on Locally Compact Abelian Groups. Springer-Verlag, New York-Heidelberg.

[5] J. Bertoin (1996), Lévy Processes. Cambridge Univ. Press.

[6] J. Bertoin (1999), Subordinators: Examples and Applications. In: Lectures on Probability Theory and Statistics (Saint-Flour, 1997), pp. 1-91, Lecture Notes in Math., 1717, Springer-Verlag, Berlin.

[7] R. M. Blumenthal and R. Getoor (1960), A dimension theorem for sample functions of stable processes. Illinois J. Math. 4, 370-375.

[8] R. M. Blumenthal and R. Getoor (1961), Sample functions of stochastic processes with stationary independent increments. J. Math. Mech. 10, 493-516.

[9] R. M. Blumenthal and R. Getoor (1968), Markov Processes and Potential Theory. Academic Press, New York.

[10] J. Bretagnolle (1971), Résultats de Kesten sur les processus à accroisements indépendants. Sém. de Prob. V, Lecture Notes in Math., 191, 21-36, SpringerVerlag, Berlin.

[11] R. C. Dalang (2003), Level sets and excursions of the Brownian sheet. In: Topics in spatial stochastic processes (Martina Franca, 2001), 167-208, Lecture Notes in Math., 1802, Springer, Berlin.

[12] R. C. Dalang and T. S. Mountford (1996), Nondifferentiability of curves on the Brownian sheet. Ann. Probab. 24, 182-195.

[13] R. C. Dalang and T. S. Mountford (1997), Points of increase of the Brownian sheet, Probab. Th. Rel. Fields, 108, 1-27.

[14] R. C. Dalang and T. Mountford (2001), Jordan curves in the level sets of additive Brownian motion. Trans. Amer. Math. Soc. 353, 3531-3545.

[15] R. C. Dalang and T. Mountford (2002), Eccentric behaviors of the Brownian sheet along lines. Ann. Probab. 30, 293-322.

[16] R. C. Dalang and T. Mountford (2003), Non-independence of excursions of the Brownian sheet and of additive Brownian motion. Trans. Amer. Math. Soc. 355, 967-985 
[17] R. C. Dalang and J. B. Walsh (1992), The sharp Markov property of Lévy sheets. Ann. Probab. 20, 591-626.

[18] R. C. Dalang and J. B. Walsh (1993a), Geography of the level sets of the Brownian sheet. Probab. Th. Rel. Fields, 96, 153-176.

[19] R. C. Dalang and J. B. Walsh (1993b), The structure of a Brownian bubble. Probab. Th. Rel. Fields, 96, 475-501.

[20] A. Dembo, Y. Peres, J. Rosen and O. Zeitouni (2000), Thick points for spatial Brownian motion: Multifractal analysis of occupation measure. Ann. Prob., 28, 135 .

[21] J. Doob (1984), Classical Potential Theory and its Probabilistic Counterpart. Springer-Verlag, Berlin.

[22] W. Ehm (1981), Sample function properties of multi-parameter stable processes. Z. Wahrsch. Verw Gebiete 56, 195-228.

[23] S. N. Evans (1987a), Multiple points in the sample paths of a Lévy process. Probab. Th. Rel. Fields 76, 359-367.

[24] S. N. Evans (1987b), Potential theory for a family of several Markov processes. Ann. Inst. Henri Poincaré Probab. Statist. 23, 499-530.

[25] K. J. Falconer(1990), Fractal Geometry - Mathematical Foundations and Applications. Wiley \& Sons, Chichester.

[26] P. J. Fitzsimmons and T. S. Salisbury (1989), Capacity and energy for multiparameter processes. Ann. Inst. Henri Poincaré Probab. Statist. 25, 325-350.

[27] B. E. Fristedt (1967), An extension of a theorem of S. J. Taylor concerning the multiple points of the symmetric stable process. Z. Wahrsch. Verw. Gebiete $\mathbf{9}, 62$ 64 .

[28] B. E. Fristedt (1974), Sample functions of stochastic processes with stationary, independent increments. Adv. in Probab. III, pp. 241-396, Dekker.

[29] B. E. Fristedt and W. E. Pruitt (1971), Lower functions for increasing random walks and subordinators. Z. Wahrsch. Verw. Gebiete 18, 167-182.

[30] J. Hawkes (1977), Local properties of some Gaussian processes. Z. Wahrsch. Verw. Gebiete 40, 309-315.

[31] J. Hawkes (1978a), Measures of Hausdorff type and stable processes. Mathematika 25, 202-210.

[32] J. Hawkes (1978b), Image and intersection sets for subordinators. J. London Math. Soc. (2) 17, 567-576.

[33] J. Hawkes (1978c), Multiple points for symmetric Lévy processes. Math. Proc. Camb. Philos. Soc. 83, 83-90.

[34] J. Hawkes (1981), Trees generated by a simple branching process. J. London Math. Soc. (2) 24, 373-384.

[35] J. Hawkes (1998), Exact capacity results for stable processes. Probab. Th. Rel. Fields 112, 1-11.

[36] W. J. Hendricks (1973), A dimension theorem for sample functions of processes with stable components. Ann. Probab. 1, 849-853.

[37] W. J. Hendricks (1974), Multiple points for a process in $\mathbb{R}^{2}$ with stable components. Z. Wahrsch. Verw. Gebiete 28, 113-128. 
[38] F. Hirsch (1995), Potential Theory related to some multiparameter processes. Potential Analysis, 4, 245-267.

[39] F. Hirsch and S. Song (1995a), Symmetric Skorohod topology on $n$-variable functions and hierarchical Markov properties of $n$-parameter processes. Prob. Th. Rel. Fields, 103(1), 25-43.

[40] F. Hirsch and S. Song (1995b), Markov properties of multiparameter processes and capacities. Prob. Th. Rel. Fields, 103(1), 45-71.

[41] J.-P. Kahane (1972), Ensembles parfaits et processus de Lévy. Period. Math. Hungar. 2, 49-59.

[42] J.-P. Kahane (1983), Points multiples des processus de Lévy symétriques stables restreints á un ensemble de valeurs du temps. Seminar on Harmonic Analysis, 1981-1982, pp. 74-105, Publ. Math. Orsay 83, 2, Univ. Paris XI, Orsay.

[43] J.-P. Kahane (1985a), Some Random Series of Functions. 2nd edition. Cambridge Univ. Press.

[44] J.-P. Kahane (1985b), Ensembles aléatoires et dimensions. In: Recent Progress in Fourier Analysis (El Escorial, 1983), pp. 65-121, North-Holland Math. Stud., 111, North-Holland, Amsterdam.

[45] R. Kaufman (1972), Measures of Hausdorff-type, and Brownian motion. Mathematika 19, 115-119.

[46] R. Kaufman (1975), Large increments of Brownian motion. Nagoya Math. J., 56, 139-145.

[47] R. Kaufman and J. M. Wu (1982), Parabolic potential theory. J. Differential Equations 43, 204-234.

[48] H. Kesten (1969), Hitting probabilities of single points for processes with stationary independent increments. Mem. Amer. Math. Soc. 93, 129 pp.

[49] D. Khoshnevisan (1995), On the distribution of bubbles of the Brownian sheet. Ann. Probab. 23, 786-805.

[50] D. Khoshnevisan (1999), Brownian sheet images and Bessel-Riesz capacity. Trans. Amer. Math. Soc. 351, 2607-2622.

[51] D. Khoshnevisan (2002), Multi-Parameter Processes: An Introduction to Random Fields. Springer-Verlag, Berlin.

[52] D. Khoshnevisan, Y. Peres and Y. Xiao (2000), Limsup random fractals. Electron. J. Probab. 5, No.5, 24 pp.

[53] D. Khoshnevisan and Z. Shi (1999), Brownian sheet and capacity. Ann. Probab. 27, $1135-1159$.

[54] D. Khoshnevisan and Z. Shi (2000), Fast sets and points for fractional Brownian motion. Séminaire de Probabilités XXXIV, pp. 393-416, Lecture Notes in Math., 1729, Springer-Verlag, Berlin.

[55] D. Khoshnevisan and Y. Xiao (2002), Level sets of additive Lévy processes. Ann. Probab. 30, 62-100.

[56] D. Khoshnevisan and Y. Xiao (2003a), Weak unimodality of finite measures, and an application to potential theory of additive Lévy processes. Proc. Amer. Math. Soc. 131, 2611-2616. 
[57] D. Khoshnevisan and Y. Xiao (2003b), Lévy processes: capacity and Hausdorff dimension. Submitted.

[58] D. Khoshnevisan, Y. Xiao and Y. Zhong (2003a), Measuring the range of an additive Lévy processes. Ann. Probab. 31, 1097-1141.

[59] D. Khoshnevisan, Y. Xiao and Y. Zhong (2003b), Local times of additive Lévy processes. Stoch. Process. Appl. 104, 193-216.

[60] J.-F. Le Gall, J. Rosen and N.-R. Shieh (1989), Multiple points of Lévy processes. Ann. Probab. 17, 503-515.

[61] R. Lyons (1990), Random walks and percolation on trees. Ann. Probab. 18, 931-958.

[62] P. Mattila (1995), Geometry of Sets and Measures in Euclidean Spaces. Cambridge Univ. Press, Cambridge.

[63] H. P. McKean, Jr. (1955), Hausdorff-Besicovitch dimension of Brownian motion paths. Duke Math. J. 22, 229-234.

[64] M. Meerschaert and Y. Xiao (2003), Dimension results for the sample paths of operator stable processes. Submitted.

[65] P. W. Millar (1971), Path behavior of processes with stationary independent increments. Z. Wahrsch. Verw. Gebiete 17, 53-73.

[66] S. Orey and S. J. Taylor (1974), How often on a Brownian path does the law of the iterated logarithm fail? Proc. London Math. Soc., 28, 174-192.

[67] R. Pemantle, Y. Peres, and J. W. Shapiro (1996), The trace of spatial Brownian motion is capacity-equivalent to the unit square. Probab. Th. Rel. Fields 106, 379399.

[68] Y. Peres (1996), Remarks on intersection-equivalence and capacity-equivalence. Ann. Inst. Henri Poincaré Phys. Théor. 64, 339-347.

[69] Y. Peres (1999), Probability on trees: an introductory climb. In: Lectures on Probability Theory and Statistics (Saint-Flour, 1997), pp. 193-280, Lecture Notes in Math., 1717, Springer-Verlag, Berlin.

[70] W. E. Pruitt (1969), The Hausdorff dimension of the range of a process with stationary independent increments. J. Math. Mech. 19, 371-378.

[71] J. Rosen (1983), A local time approach to the self-intersections of Brownian paths in space. Comm. Math. Phys. 88, 327-338.

[72] J. Rosen (1984), Self-intersections of random fields. Ann. Probab. 12, 108-119.

[73] J. Rosen (2000), Capacitary moduli for Lévy processes and intersections. Stoch. Process. Appl. 89, 269-285.

[74] K. Sato (1999), Lévy Processes and Infinitely Divisible Distributions. Cambridge Univ. Press, Cambridge.

[75] N. R. Shieh (1998), Multiple points of dilation-stable Lévy processes. Ann. Probab. 26, 1341-1355.

[76] M. L. Straf (1972), Weak convergence of stochastic processes with several parameters. In: Proc. Sixth Berkeley Sympos. Math. Statist. Probab. (Univ. California, Berkeley, Calif., 1970/1971), 2, pp. 187-221. Univ. California Press, Berkeley.

[77] S. J. Taylor (1953), The Hausdorff $\alpha$-dimensional measure of Brownian paths in n-space. Proc. Camb. Philos. Soc. 49, 31-39. 
[78] S. J. Taylor (1955), The $\alpha$-dimensional measure of the graph and set of zeros of a Brownian path. Proc. Camb. Philos. Soc. 51, 265-274.

[79] S. J. Taylor (1966), Multiple points for the sample paths of the symmetric stable process. Z. Wahrsch. Verw. Gebiete 5, 247-264.

[80] S. J. Taylor (1986a), The measure theory of random fractals. Math. Proc. Camb. Philos. Soc. 100, 383-406.

[81] S. J. Taylor (1986b), The use of packing measure in the analysis of random sets. Lecture Notes in Math., 1203, 214-222.

[82] S. J. Taylor and N. A. Watson (1985), A Hausdorff measure classification of polar sets for the heat equation. Math. Proc. Camb. Philos. Soc. 97, 325-344.

[83] M. E. Vares (1983), Local times for two-parameter Lev́y processes. Stochastic Process. Appl., 15, 59-82.

[84] J. B. Walsh (1986). Martingales with a Multidimensional Parameter and Stochastic Integrals in the Plane. (Ed's: G. del Piño and R. Robodello), Lecture Notes in Math. 1215, Springer, Berlin.

[85] N. A. Watson (1976), Green functions, potentials, and the Dirichlet problem for the heat equation. Proc. London Math. Soc. 33, 251-298; Corrigendum, ibid., 37 (1978), 32-34.

[86] N. A. Watson (1978), Thermal capacity. Proc. London Math. Soc. 37, 342-362.

[87] Y. Xiao (2003), Random fractals and Markov processes. Proceedings of the Conference on Fractal Geometry and Applications: A Jubilee of Benoit Mandelbrot, (to appear).

[88] Y. Zhong and Y. Xiao (1995), Self-intersection local times and multiple points of the stable sheet. Acta Math. Sci. (Chinese) 15, 141-152.

Davar Khoshnevisan

Department of Mathematics

155 South 1400 East JWB 233

University of Utah

Salt Lake City, UT 84112-0090

USA

e-mail: davar@math.utah.edu

URL: http://www.math.utah.edu/ davar

Yimin Xiao

Department of Statistics and Probability

A-413 Wells Hall

Michigan State University

East Lansing, MI 48824

USA

e-mail: xiao@stt.msu.edu

URL: http://www.stt.msu.edu/ ${ }^{\sim}$ xiaoyimi 